\title{
Estimation of solar disk gamma-ray emission based on Geant4
}

\author{
Zhe Li ${ }^{1,2^{*}}$, Songzhan Chen ${ }^{1,2^{* *}}$, Yuncheng $\mathrm{Nan}^{3}$, Huihai $\mathrm{He}^{1,2}, \operatorname{Cong~Li}^{1}$ \\ ${ }^{1}$ Key Laboratory of Particle Astrophyics, Institute of High Energy Physics, Chinese Academy of \\ Sciences, Beijing, 100049, China \\ ${ }^{2}$ TIANFU Cosmic Ray Research Center, Chengdu, China \\ ${ }^{3}$ Shangdong University, Qingdao, 266237, China \\ Email:"lizhe@ihep.ac.cn, speaker **chensz@ihep.ac.cn
}

\begin{abstract}
:
The most important astrophysical mechanism for steady solar gamma-ray emission is the interaction of cosmic rays with solar atmosphere, which has been definitely detected by Fermi-LAT with maximum energy up to $200 \mathrm{GeV}$. Some studies shown that the effect of the solar magnetic field to solar disk gamma-ray flux is unneglectable. It is valuable to study the physical mechanism between solar disk gamma-ray flux and solar magnetic field. In this paper, we simulated the interaction between cosmic rays and solar atmosphere by G4SOLAR program based on Geant 4 . A simulated solar disk gamma-ray flux from $1 \mathrm{GeV}$ to $1 \mathrm{TeV}$ was obtained with taking into account the influence of coronal magnetic field. The PFSS model for "quiet"Sun was employed, and the solar disk gamma-ray below $10 \mathrm{GeV}$ was consistent with the Fermi observation. But the simulated spectrum became much softer than Fermi data when $>10 \mathrm{GeV}$. Some influence factors were briefly discussed.
\end{abstract}

36th International Cosmic Ray Conference -ICRC2019

July 24th - August 1st, 2019

Madison, WI, U.S.A. 


\section{Introduction}

The most important astrophysical mechanism for steady solar gamma-ray production is the interaction of cosmic rays (CR) with solar matter and photons, that has been definitely detected by Fermi-LAT with maximum energy up to $200 \mathrm{GeV}$ (Abdo et al. 2011; $\mathrm{Ng}$ et al. 2016; Tang et al. 2018). The gamma-ray emission from the solar disk due to $\mathrm{CR}$ cascades in the solar atmosphere is denoted as disk component, and its luminosity is correlated both with the flux of primary cosmic rays and the intensity of solar magnetic field.

Some experiments have observed the solar disk gamma-ray spectrum or upper limit during different solar activity period. Kenny, et al (2016) firstly observated the time variation in the solar-disk gamma-ray flux with Fermi up to $100 \mathrm{GeV}$. In the 1-10 GeV flux they discovered a significant time variation that anticorrelates with solar activity. Tang, et al (2018) analyzed 9 years of Fermi data up to $200 \mathrm{GeV}$ to detect the solar-disk gamma-ray flux and study its spectrum and time variation. The moderate time variations is about $\pm 50 \%$ in the solar disk gamma-ray flux between $1-10 \mathrm{GeV}$, and the amplitude of this variation does not seem to vary significantly with energy. They also find that the observed solar disk gamma-ray flux has a significantly harder spectrum $\left(\sim \mathrm{E}^{-2.2}\right)$, relative to the cosmic-ray spectrum $\left(\sim \mathrm{E}^{-2.7}\right)$. HAWC observation searched for 1-100 $\mathrm{TeV}$ gamma rays from the solar disk by using data from November 2014 to December 2017, just prior to the solar minimum. No evidence of a signal is observed, and they set strong upper limits on the flux at a few $10^{-12} \mathrm{TeV}^{-1} \mathrm{~cm}^{-2} \mathrm{~s}^{-1}$ at $1 \mathrm{TeV}$ (Albert, et al.,2018). ARGO-YBJ experiment gave the upper limits to the gamma-ray flux at $0.3-7 \mathrm{TeV}$ at $95 \%$ confidence level during solar minimum (Bartoli, et al., 2019).

The solar disk gamma-ray expectation or simulation also achieved some important results. The upper limit of $20 \mathrm{KeV}-10 \mathrm{MeV}$ solar gamma-ray was obtained by Dolan et al., (Dolan et al. 1965; Peterson et al. 1966). A detailed theoretical model (SSG model) for gamma-ray from the collision of cosmic ray with the solar atmosphere was presented by Seckel et al. (1991). The predicted gamma-ray flux at energies from $10 \mathrm{MeV}$ to $10 \mathrm{GeV}$ has a large uncertainty, being sensitive to the assumptions about the cosmic-ray transport in the magnetic field near the Sun. Recently (Zhou et al. 2017; Gao et al, 2018) have estimated the solar disk gamma-ray flux up to $100 \mathrm{TeV}$ without taking into account the effect of the solar magnetic field. The predicted flux is at least one order of magnitude lower than that measured by Fermi-LAT.

In this paper, we investigated a G4SOLAR program based on Geant4 to simulate the hardronic interaction between cosmic ray (e.t. proton) and solar atmosphere. The coronal magnetic field model of PFSS was adopted to take into account the influence of the coronal magnetic field.

\section{G4SOLAR simulation}


The main Monte Carlo simulation process is: generate a proton as the primary cosmic ray particle with random position, direction and energy $(1 \mathrm{GeV}-100 \mathrm{TeV})$; then this primary proton particle will propagate into the inner solar atmosphere and generate hardronic shower; the production of gamma-ray above $1 \mathrm{GeV}$ will be recored by Geant4.

The same size of solar ( $\mathrm{R}=700000 \mathrm{~km})$ was constructed in G4SOLAR program. Bei et al. (2018) obtained the solar mass density above the photosphere and optical depth for inelastic proton-proton collisions. This result was adopted here to set the inner and outer height of photosphere, $600 \mathrm{~km}$ and $3000 \mathrm{~km}$, respectively. We divided the $3600 \mathrm{~km}$ solar atmosphere into 3600 layers with a thickness of $1 \mathrm{~km}$ for each layer. In inner layers, the solar matter (proton) density was given by SSG (Seckel et al.,1991). In outer layers, the density shown in Marvin L White et al.,(1966) was adopted here for inner 1600 layers, and the density for outer 1400 layers was filled by the results of function 2.1 in Seckel et al.,(1991).

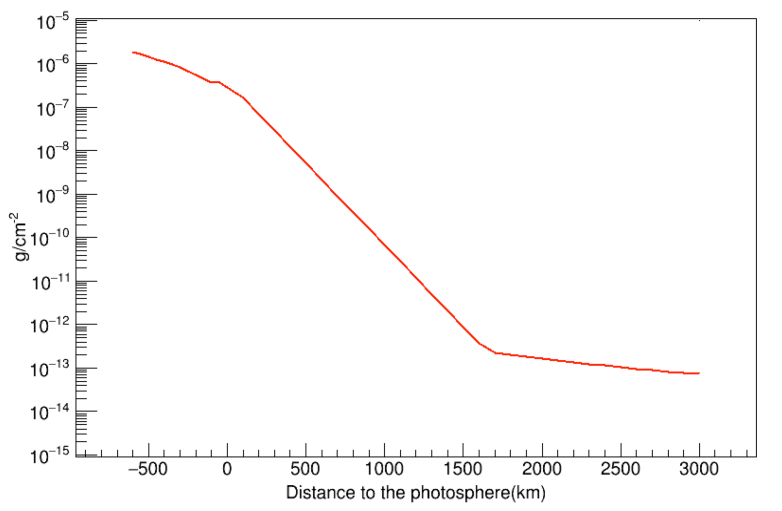

Figure 1. The solar atmosphere density used in G4SOLAR

The interaction between cosmic ray and solar atmosphere will be more complicated with the existence of solar magnetic field. In this G4SOLAR program, we adopted a solar magnetic field model for a "quiet" Sun. The potential-field source-surface (PFSS), proposed by Schatten (1969), was used in G4SOLAR. In this PFSS model, the magnetic field construction was resolved by the interpolation of observed coronal magnetic field structure during 2018y. The radial magnetic field at photosphere surface is shown in figure 2.

As the solar magnetic field is non-uniform, the incident position of primary proton must be distributed in the whole solar surface uniformly, and the incident direction $\omega$, the included angle between normal direction and incident direction, should consider the direction projection to the surface. Only when $90^{\circ}<\omega<180^{\circ}$ the primary proton can enter into the solar atmosphere, the distribution of sampling $\omega$ is shown in Fig.3 left. From the Fig.3 right, we can get that the production of gamma-ray are mainly produced by the proton with $95^{\circ}<\omega<100^{\circ}$, this result is consistent with the result given by fixed incident position simulation (Gao et al., 2018). 


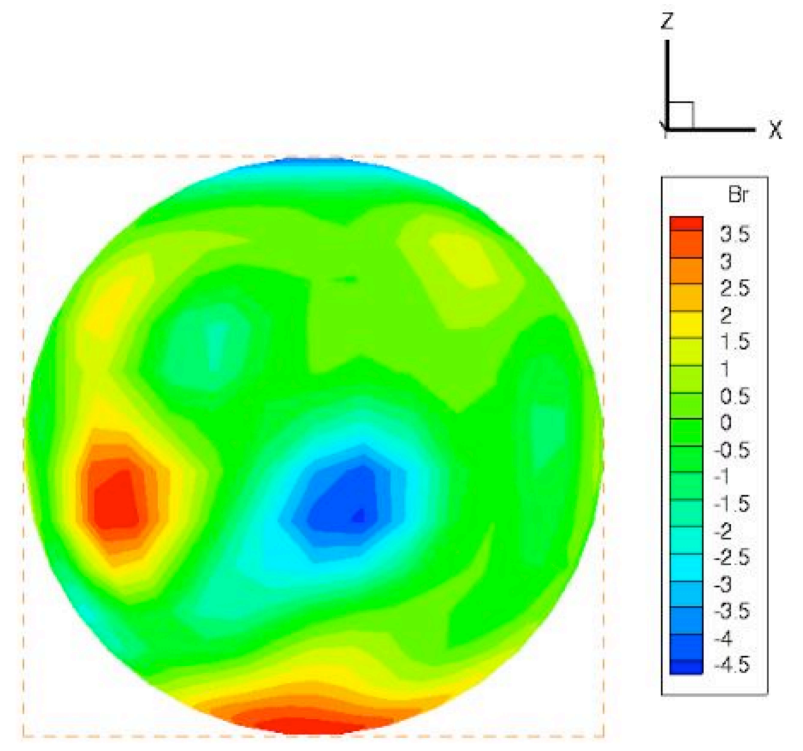

Figure 2. Radial magnetic field at photosphere surface of PFSS model for "quiet" Sun
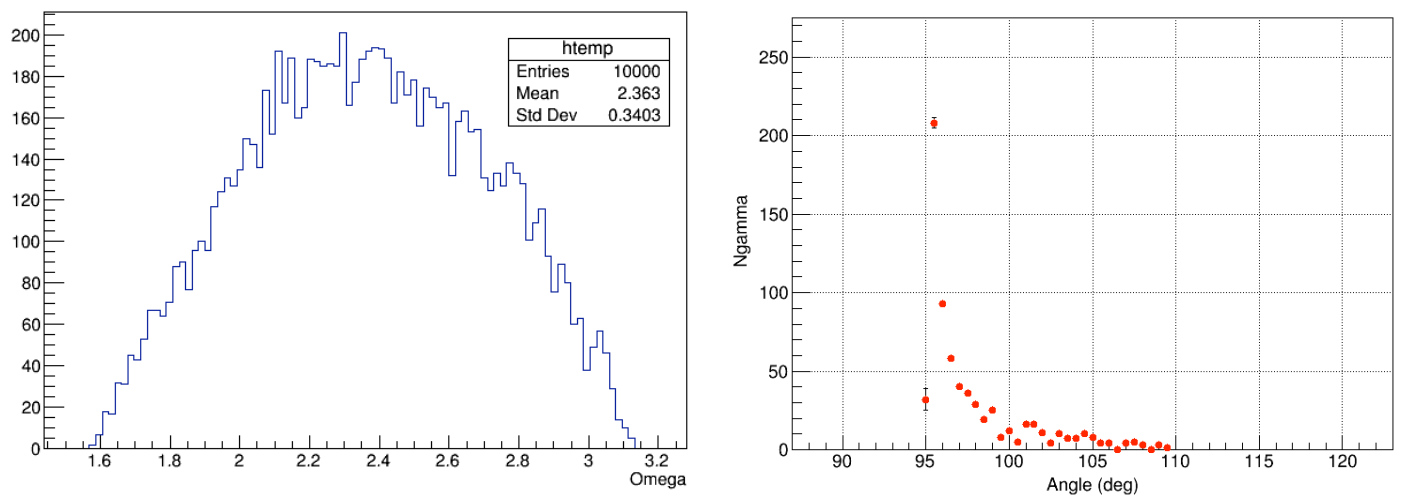

Figure 3. left: the incident direction distribution of primary particle; right: the induced gamma number vs. the incident direction $\omega$.

\section{Solar disk simulation result}

The solar disk gamma-ray comes from photons produced in the solar cascade. These may arise either from two proton decays of $\pi$ 's or from the electromagnetic shower in the latter part of the cascade. The FTFP_BERT physics interaction lib was employed in the G4SOLAR program. This lib contains all standard electromagnetic process, and the Bertini cascade is used for hadron $<5 \mathrm{GeV}$, Fritiof model for hadron $>4 \mathrm{GeV}$.

In order to check the performance of G4SOLAR program, we firstly get the solar disk gamma-ray spectrum with no magnetic field, as shown in Fig.4. The result is consistent with Gao's result, which are much higher than Bei's result. It is mainly because the incident direction of primary proton was sampled from $0^{\circ} \sim 180^{\circ}$ for zenith angle, which is larger than that used in other simulation results.

When the coronal magnetic field PFSS model for "quiet" Sun put into the G4SOLAR program, the hardronic interaction process will be more complicated. 
There are mainly two cases for charged particles during the interaction process. Particles with low energy will escape the solar limb very fast with decreasing the times of physics interaction. This process lead the results of fewer gamma-ray can be produced. But for particles with high energies, their motion path can be deflected, and effective interaction with solar atmosphere will be increased, thus leads to more gamma-ray can be produced, especially for gamma-ray at lower energy range. Hence, the observed gamma-ray flux should have been significantly boosted by the solar magnetic field and the spectrum would change around the critical energy.

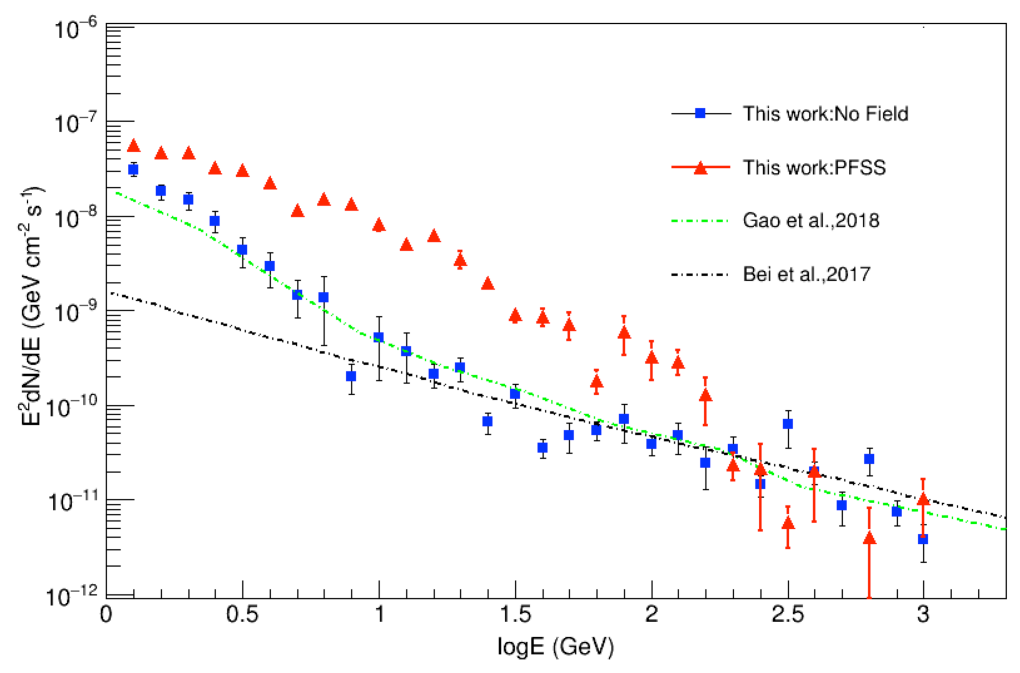

Figure 4. The simulation results of solar disk gamma-ray spectrum

The simulation results of solar disk gamma-ray spectrum with PFSS model for "quiet" Sun is shown in Fig.4. The gamma-ray energy is from $1 \mathrm{GeV}$ to $1 \mathrm{TeV}$, and the number for gamma rays at lower energy are obviously increased as compared with no-field result. This result met the expectation of proliferative effect caused by the corona magnetic field. We also can observed that there is no proliferative result for gamma-ray $>150 \mathrm{GeV}$, which implied that the coronal magnetic field make little effect for high energy solar disk gamma rays.

\section{Summary}

A very preliminary result of solar disk gamma-ray simulation with PFSS model for "quiet" Sun was obtained. The spectrum index is much harder than that with no magnetic field, but much softer than the observed result obtained by Fermi during solar minimum period, shown in Fig.5. For solar disk gamma-ray $<10 \mathrm{GeV}$, the spectrum is consist with Fermi data within the margin of error. But for gamma-ray $>10 \mathrm{GeV}$, the simulated spectrum became softer very quickly than Fermi data.

Some of the factors should be briefly discussed here. The cosmic ray spectrum we put into the G4SOLAR program is that observed near the earth, and only the component of proton was considered. The Fermi data was observed during the solar minimum from 2008-8-7 to 2010-1-21. The coronal magnetic field used in 
G4SOLAR is the interpolation results of observed coronal magnetic field structure during the year 2018. The different magnetic field structure can lead to the difference of solar disk gamma-ray spectrum. The simulation range for solar atmosphere is only $3600 \mathrm{~km}$ in total, which may also affect the gamma-ray production.

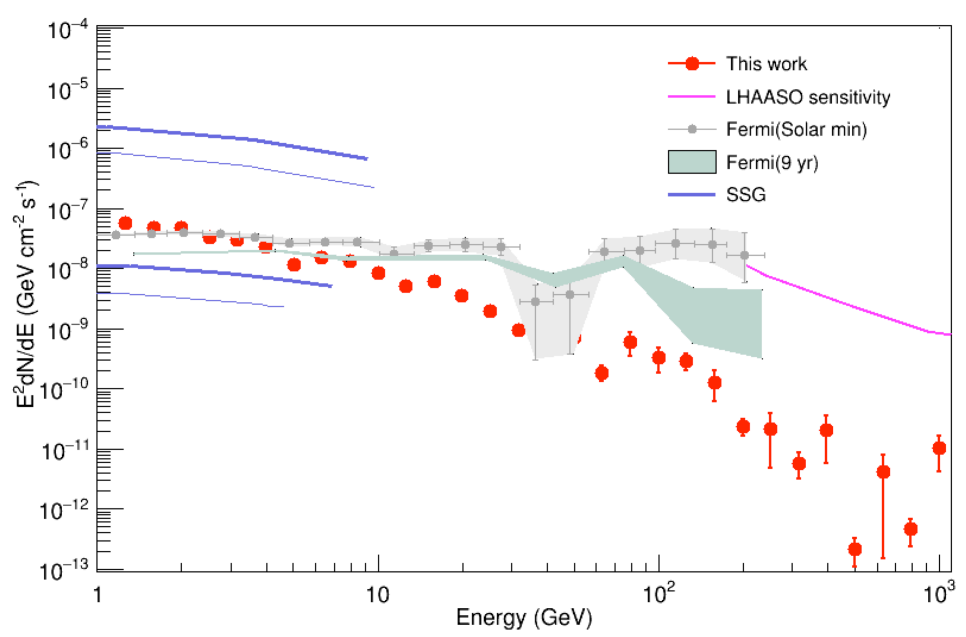

Figure 5. The G4SOLAR simulation result comparison with Fermi data (Tang et al.,2018). The sensitivity of LHAASO observatory and SSG model result are also shown in the figure.

In the future, more accurate simulation will be considered, such as PFSS model for "active" solar, more cosmic ray component to be simulated (e.g. $\mathrm{He}, \mathrm{CNO}, \mathrm{MgAlSi}$, $\mathrm{Fe}$ ), and invers Compton simulation for the IC component. These simulations should be valuable to study the solar neutrino, dark matter model and electron spectrum.

\section{Acknowledgement}

This work is supported by the National Key R\&D Program of China (No. 2018YFA0404201) and the Natural Sciences Foundation of China (No. 11575203, 11635011).

\section{References}

[1.] Abdo, A. A., Ackermann, M., Ajello, M., et al. 2011, ApJ, 734, 116

[2.] Bartoli, B., et al., for the ARGO_YBJ collatbration, 2019,APJ,872:143

[3.] Dolan, J. F., \& Fazio, G. G. 1965, RvGSP, 3, 319

[4.] Gao B.S., Study of solar disk gamma rays based on Geant4, 2018 (in Chinese).

[5.] Ng, K. C. Y., Beacom, J. F., Peter, A. H. G., \& Rott, C. 2016, PhRvD, 94, 023004

[6.] Seckel, D., Stanev, T., \& Gaisser, T. K. 1991, ApJ, 382, 652

[7.] Tang, Q. W., Ng, K. C. Y., Linden, T., et al. 2018, PhRvD, 98, 063019

[8.] Albert, A., Alfaro, R., Alvarez, C., et al. 2018a, PhRvD, 98, 123011

[9.] Zhou, B., Ng, K. C. Y., Beacom, J. F., \& Peter, A. H. G. 2017, PhRvD, 96, 023015 